\title{
CARACTERIZAÇÃO E DINÂMICA DE DUAS FASES SUCESSIONAIS EM FLORESTA SECUNDÁRIA DA MATA ATLÂNTICA ${ }^{1}$
}

\author{
Ademir Roberto Ruschel ${ }^{2}$, Marcelo Mantovani ${ }^{3}$, Maurício Sedrez dos Reis ${ }^{4}$ e Rubens Onofre Nodari ${ }^{4}$
}

\begin{abstract}
RESUMO - Em Santa Catarina, foi observado aumento da cobertura florestal nas últimas décadas, o que vem construindo uma paisagem florestal retalhada por um grande mosaico de fragmentos de vários estádios sucessionais. Neste trabalho, buscou-se avaliar o dinamismo do processo sucessional de dois diferentes estádios sucessionais. Em área florestal de 40 ha localizada no Município de São Pedro de Alcântara, SC, abandonada pelo uso agropecuário em meados de 1970, foram estabelecidas aleatoriamente parcelas permanentes (50 x $50 \mathrm{~m})$, duas em estádio florestal secundário médio (SM) e quatro em estádio secundário avançado (SA). As avaliações anuais durante o período de 1994 a 2000 de todas as plantas arbóreas com DAP $\geq 5 \mathrm{~cm}$ revelaram que no SM os valores da densidade de plantas, residentes, recrutadas, mortas e ramificadas foram superiores em relação aos no SA. No entanto, a riqueza de espécies, área basal e distribuição diamétrica foram superiores no SA. Botanicamente, foram observadas com muita clareza as espécies e as famílias dominantes de cada estádio e igualmente o dinamismo sucessional desse grupo de espécies, aumento explosivo e posterior declínio e substituição, evidenciando-se perfeitamente a funcionalidade dos grupos ecológicos nessa tipologia florestal. Por fim, destacou-se que a densidade de plantas ramificadas é notadamente superior em estádios florestais secundários iniciais, embora as taxas de incremento corrente anual fossem similares. As avaliações florísticas mostraram, ainda, que as florestas no litoral catarinense se encontravam em dinâmica sucessional, em que espécies climáxicas vêm substituindo paulatinamente o grupo de espécies pioneiras, elevando a diversidade de espécies e a biomassa florestal.
\end{abstract}

Palavras-chave: Espécies florestais, incremento diamétrico, mortalidade, ramificação e recrutamento.

\section{CHARACTERIZATION AND DYNAMICS OF TWO SUCCESSIONAL STAGES OF SECONDARY ATLANTIC FOREST}

\begin{abstract}
In Santa Catarina State, the forested area of the Atlantic Forest increased in the last decade, forming a landscape which is characterized by a mosaic of fragments of distinct successional stages. This work aimed to evaluate the successional process dynamics of two successional stages in the secondary Atlantic forest. This study was carried out in a secondary forest remnant near São Pedro de Alcântara-SC (area of $40 \mathrm{ha})$, abandoned since 1970 . Permanent plots $(50 \times 50 \mathrm{~m})$ were established randomly, two in a secondary medium growth (SM) and four in a secondary old growth (SA) forest stages. The annual evaluations from 1994 to 2000 on all trees with $D B H \geq 5 \mathrm{~cm}$ revealed that while the SM stage exhibited superior values for density, recruited, dead and branched plants, the SA stage exhibited superior values for species richness, basal area and diametric class. Botanically, the dominant species and families in each stage were clearly observed, as well as the succession dynamics of this species group, explosive increase and posterior decrease and substitution, perfectly demonstrating the functionality of the ecological groups of this forest typology.
\end{abstract}

\footnotetext{
${ }^{1}$ Recebido em 21.02.2007 e aceito para publicação em 26.01.2009.

${ }^{2}$ Empresa Brasileira de Pesquisa Agropecuária (EMBRAPA), Belém-PA. E-mail: <ruschel@ @patu.embrapa.br>.

${ }^{3}$ Engenheiro Agrônomo, Prefeitura de Joaçaba, SC. E-mail: <marcelomanto@ yahoo.com>.

${ }^{4}$ Departamento de Fitotecnia da Universidade Federal de Santa Catarina (UFSC). E-mail: <msreis@ @ca.ufsc.br>e <nodari@ cca.ufsc.br>.
} 
Finally, the density of the plants branched was found to be superior at the initial secondary forest stages, although the rates of annual current increase were similar. The floristic evaluations also showed that the forests in the Santa Catarina coast were in a succession dynamics, where pioneer species have been gradually replaced by climax species, increasing species diversity and forest biomass.

Keywords: Diameter increment, forest species, mortality, ramification and recruitment.

\section{INTRODUÇÃO}

No Estado de Santa Catarina ocorrem três tipologias florestais que pertencem ao megacossistema Mata Atlântica (IBGE, 1990). Entre essas, a tipologia florestal da encosta litorânea, a que Klein (1978) denominava Floresta Pluvial da Encosta Atlântica ou Floresta Ombrófila Densa. Essa tipologia foi intensamente reduzida e alterada pelo uso agropecuário e industrial, restando aproximadamente 7\% da área original (Fundação SOS Mata Atlântica e INPE, 2008).

No Relatório da Fundação SOS Mata Atlântica e INPE (2008) foi constatada, no Estado de Santa Catarina, uma diminuição de $7 \%$ nas taxas de desmatamento em relação ao período anterior (19952000), de modo que, se somadas todas áreas de formações florestais secundárias em diferentes idades e remanescentes de floresta primária alteradas, conjuntamente representam aproximadamente $24 \%$ da cobertura original. Tal realidade foi decorrente, em parte, pelo abandono de atividades agropecuárias, estas principalmente em áreas íngremes e em parte pela eficácia e rigor da legislação florestal que proíbe a derrubada de capoeiras nos estádios médio e avançado de sucessão (CONAMA, 1994), o que atingiu o sistema de rotação coivara e pousio. Esse cenário proporcionou o surgimento da paisagem florestal constituída por mosaicos de vegetação em vários estádios sucessionais (QUEIROZ, 1994).

Steenis (1958) introduziu as primeiras discussões sobre dinamismo florestal (conceito espécies nômades), e, posteriormente, Budowski $(1965,1970)$ classificou as espécies florestais em grupos ecológicos ou grupos sucessionais, conforme as características que estas apresentam.

A dinâmica sucessional em florestas pode ser caracterizada principalmente pelas mudanças na flora e na fauna decorrentes em determinado período. Em tese, floristicamente a dinâmica sucessional pode ser mensurada pela entrada (recrutamento), saída (mortalidade) e crescimento das espécies que participam da estrutura florestal. As mudanças florísticas provocadas pela mortalidade e pelo recrutamento de plantas podem ser decorrentes de vários fatores: predação/herbivoria, competição espacial, doenças e perturbações naturais. Tais fatores são importantes para a dinâmica de ecossistemas florestais, pois geram heterogeneidade ambiental que contribuem para o aumento da diversidade das espécies ou, ainda, são bases para a reprodução, recrutamento, especialização e partilha de recursos (BUDOWSKI, 1965, 1970; JANZEN, 1970; WHITMORE, 1983; TILMAN, 1994; TABARELLI e MANTOVANI, 1997; DALLING et al., 1998).

As primeiras descrições sobre dinamismo florístico em formações florestais secundárias da Floresta Ombrófila Densa no Sul do Brasil foram abordadas por Klein (1980). Contudo, estudos sobre dinamismo dentro da comunidade florística, avaliados em diferentes intervalos de tempos, são poucos, e a maioria destes foi realizada no Sudeste e Norte do Brasil (PAGANO et al., 1995; OLIVEIRA-FILHO et al., 1997; FERREIRA et al., 1998; NASCIMENTO et al., 1999; WERNECK et al., 2000).

Inventários fitossociológicos e o monitoramento em parcelas permanentes com abordagens quantitativas (diversidade e índices de riqueza de espécies, abundância e dominância, entre outros) auxiliam a compreensão da complexidade estrutural de uma comunidade florestal (MAGURRAN, 1988). Apesar das limitações ecológicas desses índices, os modelos matemáticos são atualmente as abordagens mais usadas. Recentemente, Petrere et al. (2004) usaram a abordagem quantitativa em estudos fitossociológicos para a compreensão do estádio sucessional de uma formação vegetacional.

Levando-se em consideração, portanto, que a cobertura florestal catarinense é constituída principalmente por mosaicos de florestas secundárias, é nítida a necessidade de maior conhecimento sobre a dinâmica e florística desses remanescentes, com vistas a proporcionar bases e diretrizes para definir estratégias de uso, restauração e conservação deles. Dessa forma, neste trabalho se buscou caracterizar o dinamismo do 
processo sucessional pelos aspectos florísticos e dendrométricos em dois diferentes estádios sucessionais secundários da Floresta Ombrófila Densa no Sul do Brasil.

\section{MATERIAL E MÉTODO}

A área de estudo localiza-se no Município de São Pedro de Alcântara, SC (MANTOVANI et al., 2003, 2005). A vegetação original do local, segundo Klein (1978, 1980), é classificada de Floresta Ombrófila Densa. A área de estudo compreende dois estádios sucessionais que foram denominados: secundário médio ( $\mathrm{SM}$ ), este caracteriza o estádio com menor tempo de recuperação em relação ao segundo estádio, aqui denominado secundário avançado (SA). O SM refere-se à área em que foram abandonadas as atividades agropecuárias no início de 1970; e secundário avançado, área que sofreu exploração intensiva há mais de cinco décadas antes da primeira avaliação, principalmente das espécies de valor madeireiro. As avaliações foram feitas em seis parcelas permanentes $(50 \times 50 \mathrm{~m})$, estabelecidas aleatoriamente. Quatro parcelas (A, B, C e D) encontramse em sucessão SA e as parcelas E e F, em sucessão SM.

Foram feitas avaliações anuais no período de 19942000, nos meses de janeiro e fevereiro. Cada planta arbustivo-arbórea residente (excluídos espécimes das famílias Cyatheacea e Arecaceae) com DAP $\geq 5 \mathrm{~cm}$ (diâmetro a altura do peito, 1,3 m ) foi identificada com etiqueta, mapeada, medido o DAP (paquímetro florestal com escala milimétrica) e identificada por família e espécie. A identificação das espécies foi feita in loco quando inequívoca. Com as espécies desconhecidas, procedeuse à coleta do material botânico, e posteriormente elas foram identificadas com o auxílio de bibliografia especializada e profissionais da área.

A distribuição diamétrica das espécies foi agrupada em intervalos de $10 \mathrm{~cm}$ de DAP, permitindo, assim, caracterizar e comparar a distribuição diamétrica por espécie e entre os dois estádios sucessionais.

Foram classificadas como planta ramificada todas as plantas que apresentavam ramificação do tronco principal na altura inferior ao DAP e como plantas remanescentes, toda planta viva com DAP $\geq 5 \mathrm{~cm}$ mensurada no ano da avaliação. Cada planta ramificada foi considerada como apenas um indivíduo remanescente, embora todo ramo com DAP $\geq 5 \mathrm{~cm}$ foi incluído nas estimativas de área basal e incremento corrente anual. Para a análise da densidade e distribuição diamétrica, apenas o ramo de maior diâmetro foi levado em consideração. Foi considerada planta recrutada toda planta que, no ano da avaliação, apresentou DAP $\geq 5 \mathrm{~cm}$ e que não havia atingido ainda esse diâmetro no ano anterior. Por planta morta, enquadrou-se toda planta avaliada num ano e ausente ou morta no ano seguinte. As taxas de mortalidade e recrutamento foram calculadas em porcentagem, sendo a taxa a simples proporção do número de plantas mortas no período analisado em relação ao número de plantas inicialmente avaliadas (primeira medição). A mesma lógica foi usada para plantas recrutadas.

Nas análises da similaridade entre as fases SM e SA, usaram-se os índices de diversidade Shannon e Wiener (acompanhado do teste-t), equabilidade de Shannon, índices de similaridade Jaccard, conforme Magurran (1988), e índice de similaridade Bray-Curtis, de acordo com Krebs (1989). Adicionalmente às comparações para testar a distribuição das espécies, usou-se o teste Qui-quadrado pela tabela de contingência (BEIGUELMAN, 1991).

Do DAP de cada planta avaliada foram calculados os valores de área basal, DAP médio e o desvio-padrão da média. Também foi calculado o incremento corrente anual (ICA) por planta (FERREIRA et al., 1998). O teste-t foi utilizado para avaliar estatisticamente a distribuição anual do número médio de plantas remanescentes, recrutadas, mortas, ramificadas e DAP médio. Já nas comparações sobre a distribuição do número total de plantas ha ${ }^{-1}$ por espécie dentro dessas mesmas categorias (plantas remanescentes, recrutadas, mortas e ramificadas), entre estádios sucessionais, aplicou-se o teste Qui-quadradro.

\section{RESULTADOS E DISCUSSÕES}

A diversidade de espécies foi alta em ambos os remanescentes florestais secundários. Na formação secundário avançado (SA) foram identificadas 112 espécies e na formação secundário médio (SM), 81 espécies, valor aproximadamente $30 \%$ inferior ao estádio SA. Verificou-se que 63 espécies foram comuns aos dois estádios, 49 espécies foram exclusivas ao SA e 18 ao SM. Durante o período 1994-2000, observouse o recrutamento de três espécies novas no SA e apenas uma no SM (Tabela 1). Mantovani et al. (2003; 2005) observaram na área de estudo 133 espécies florestais, e a densidade destas distribuí-se em 59,6\% de espécies

R. Árvore, Viçosa-MG, v.33, n.1, p.101-115, 2009 
arbóreas (130 espécies), 22,3\% Cyatheaceas (duas espécies) e 18,1\% Palmae (uma espécie).

A diversidade de espécies estimadas pelo índice de Shannon ( $\mathrm{H}^{\prime}$ ) em SA foi de 3,96 no ano de 1994 e de 4,0 no ano 2000 (Tabela 1). Quando comparados aos valores encontrados na formação $S M$, eles foram significativamente diferentes $(\mathrm{P}<0,001)$. Pela equabilidade do índice de Shannon, foi estimado que a diversidade em SA foi 7\% superior, comparativamente a SM. A análise de similaridade entre os dois estádios sucessionais, indicada pelos índices de Jaccard e Bray-Curtis, detectaram altos valores de similaridade para a comunidade florística dentro do mesmo estádio sucessional durante o período avaliado, em ambos os estádios SM e SA. Contrariamente, a comparação entre os remanescentes SA e SM produziu valores extremamente baixos para esses mesmos índices de similaridade.

A densidade média de plantas remanescentes no período de sete anos em SM foi de 1.779 plantas ha ${ }^{-1}$, aproximadamente $65 \%$ superior ao estádio SA (Tabela 2), que foi de 1050. No mesmo período, a dinâmica da entrada (plantas recrutadas) e saída de plantas (plantas mortas) nas formações SA e SM ocorreu com taxas médias anuais para plantas recrutadas de $1,8 \%$ e $2,5 \%$ e para plantas mortas de $1,7 \%$ e $2,1 \%$, respectivamente. $\mathrm{O}$ balanço final entre plantas recrutadas e plantas mortas no período de 1994-2000 resultou no incremento médio anual de 1,8 planta ha ${ }^{-1}$ em SA e 6,0 plantas ha ${ }^{-1}$ em SM. Taxas de mortalidade de $2,1 \%$ (SM) e $1,7 \%$ (SA) estão dentro do esperado, pois em florestas naturais tais valores têm variado de 1 a 2\% (PHILLIPS et al., 1998; ROLIM et al., 1999; WERNECK et al., 2000).

Os valores do número médio anual de plantas recrutadas e mortas foram superiores em SM em pelo menos duas vezes, comparativamente a SA (Tabela 2). Já no caso de plantas ramificadas essa diferença foi praticamente sete vezes superior ( $32 \mathrm{em} \mathrm{SA} \mathrm{e} 220 \mathrm{em}$ $\mathrm{SM})$. Tais diferenças foram estatisticamente significativas para plantas mortas e ramificadas $(\mathrm{P}<0,001)$. O número médio de ramos nas plantas ramificadas foi 1,7 em SA e 1,8 em SM. É relevante mencionar que o número de plantas ramificadas manteve-se inalterado durante os seis anos de avaliação. Embora em ambos os estádios SA e SM se observasse também alta dinâmica no recrutamento (respectivamente 4,0 e 3,9 ramos ha/ano) e na mortalidade (respectivamente 4,9 e 5,1 ramos ha/ano), verificou-se a redução de ramos em ambos os estágios, porém ainda o número de plantas ramificadas se manteve inalterado por todo o período avaliado.

O dinamismo sucessional superior (entrada e saída de plantas) no SM foi proporcionado principalmente pelas espécies pioneiras das famílias Melastomataceas (Miconia cabucu e M. Cinnamomifolia) Euphorbiaceae (Hyeronima alchorneoides) e Asteraceae (Vernomia discolor), as quais proporcionaram alta densidade florestal. Igualmente, na Floresta Estacional Semidecidual no Estado de São Paulo foram identificadas maior densidade de plantas e menor área basal nas áreas com floresta em estádio menos avançado, em comparação com áreas de estádio mais avançado (FONSECA e RODRIGUES, 2000).

Tabela 1 - Número de espécies, índice de diversidade Shannon $\left(\mathrm{H}^{\prime}\right)$, equabilidade de Shannon (E), índices de similaridade Jaccard e Bray-Curtis e probabilidades Qui-quadrado $(\chi 2)$ para a distribuição da densidade de plantas por espécies. Dados coletados no período de 1994-2000, nos estádios sucessionais secundário avançado (SA) e secundário médio (SM), remanescentes da Floresta Ombrófila Densa em São Pedro de Alcântara, SC, Brasil

Table 1 -Number of species, Shannon diversity index ( $\left.H^{\prime}\right)$, evenness Shannon index (E), Jaccard and Bray-Curtis similarity indices, and Qui-Square $\left(\chi^{2}\right)$ probabilities for plant density per species. Data from successional secondary old growth (SA) and secondary medium growth (SM) of the Ombrophylus Dense Forest, evaluated from 1994-2000. São Pedro de Alcântara, SC, Brazil

\begin{tabular}{|c|c|c|c|c|c|}
\hline \multirow[t]{2}{*}{ Índices de diversidade } & \multicolumn{2}{|c|}{ SA } & \multicolumn{2}{|c|}{ SM } & \multirow{2}{*}{$\frac{\mathrm{SA} \times \mathrm{SM}}{2000}$} \\
\hline & & 2000 & 199 & 2000 & \\
\hline $\mathrm{N}^{\circ}$ espécies & 109 & 112 & 80 & 81 & $\mathrm{t}=11,98(\mathrm{GL}=1981)$ \\
\hline $\mathrm{H}^{\prime}$ Shannon & 3,96 & 4,00 & 3,41 & 3,42 & $\mathrm{P}<0,001$ \\
\hline E Shannon & 0,85 & 0,85 & 0,78 & 0,78 & \\
\hline Jaccard & 0,96 & 0,92 & 0,45 & & \\
\hline Bray-Curtis & \multicolumn{2}{|c|}{0,98} & \multicolumn{2}{|c|}{0,94} & 0,24 \\
\hline$\chi^{2}$ & \multicolumn{2}{|c|}{$\begin{array}{c}\mathrm{P}>0,995 \\
\text { GL } 110 ; \chi^{2}=12,7\end{array}$} & \multicolumn{2}{|c|}{$\begin{aligned} & P>0,995 \\
\text { GL } & 83, \chi^{2}=55,2\end{aligned}$} & $\begin{array}{c}\mathrm{P}<0,001 \\
\text { GL } 129, \chi^{2}=1957\end{array}$ \\
\hline
\end{tabular}

R. Árvore, Viçosa-MG, v.33, n.1, p.101-115, 2009 
Tabela 2 - Média de plantas $\left(\right.$ ha $\left.^{-1}\right)$ das categorias de remanescentes (PREM), recrutadas (PREC), mortas (PMOR) e ramificadas (PRAM), teste-t e proporção de cada categoria em relação ao total de plantas nos estádios sucessionais secundário avançado (SA) e secundário médio (SM), remanescentes da Floresta Ombrófila Densa, avaliados no período de 1994-2000. São Pedro de Alcântara, SC, Brasil

Table 2 - Means of plants $\left(\mathrm{ha}^{-1}\right)$ of remnant (PREM), recruited (PREC), dead (PMOR) and branched (PRAM) categories, $t$-test and proportion of each category related to the total of plants in the forest successional secondary old growth (SA) and secondary medium growth (SM) stages of the Ombrophylus Dense Forest, evaluated from 1994-2000. São Pedro de Alcântara, SC, Brazil

\begin{tabular}{|c|c|c|c|c|c|c|c|c|}
\hline \multirow[t]{2}{*}{ Ano } & \multicolumn{2}{|c|}{$\operatorname{PREM}\left(\mathrm{ha}^{-1}\right)$} & \multicolumn{2}{|c|}{$\operatorname{PREC}\left(\mathrm{ha}^{-1}\right)$} & \multicolumn{2}{|c|}{$\operatorname{PMOR}\left(\mathrm{ha}^{-1}\right)$} & \multicolumn{2}{|c|}{$\operatorname{PRAM}\left(\mathrm{ha}^{-1}\right)$} \\
\hline & SA & SM & SA & SM & SA & SM & SA & SM \\
\hline 1994 & 1042 & 1728 & - & - & - & - & $32(49)^{1}$ & $220(378)$ \\
\hline 1995 & 1050 & 1774 & 39 & 102 & 31 & 56 & $32(50)$ & $220(386)$ \\
\hline 1996 & 1044 & 1804 & 16 & 76 & 22 & 46 & $32(52)$ & $220(392)$ \\
\hline 1997 & 1049 & 1798 & 8 & 16 & 3 & 22 & $32(52)$ & $220(394)$ \\
\hline 1998 & 1056 & 1782 & 18 & 8 & 11 & 24 & $32(58)$ & 220 \\
\hline 1999 & 1059 & 1794 & 20 & 48 & 17 & 36 & $32(61)$ & $220(410)$ \\
\hline 2000 & 1053 & 1776 & 13 & 22 & 19 & 40 & $32(57)$ & $220(402)$ \\
\hline média & 1050 & 1779 & 19 & 45,3 & 17,2 & 37,3 & $32(54,1)$ & $220(394,3)$ \\
\hline \multicolumn{3}{|l|}{ sobre PREM (\%) } & $1,8 \%$ & $2,5 \%$ & $1,7 \%$ & $2,1 \%$ & $3,0 \%(5,2)$ & $12,4 \%(22,2)$ \\
\hline \multicolumn{3}{|l|}{ teste-t } & \multicolumn{2}{|c|}{$1,7^{\mathrm{ns}}$} & \multicolumn{2}{|c|}{$3,1^{* * *}$} & \multicolumn{2}{|c|}{$91,9^{* *}$} \\
\hline \multicolumn{5}{|c|}{ Média de ramos por PRAM } & & & 1,69 & 1,79 \\
\hline
\end{tabular}

${ }^{1}$ Total de ramos por hectare.

ns Não-significativo $\mathrm{P}>0,05 ; *$ Signific. $\mathrm{P}<0,05$; e ** Signific. $\mathrm{P}<0,001$.

Em cada um dos estádios sucessionais no intervalo de seis anos foram recrutadas 41 espécies. Entretanto, as espécies recrutadas nos dois estádios SA (área, 1 ha) e SM (área, 1/2 ha) totalizaram 65 espécies, das quais 15 foram comuns em ambos os estádios sucessionais (Tabela 3). As famílias que apresentaram maior número de espécies recrutadas em SM foram, respectivamente, Rubiaceae, Melastomataceae, Sapindaceae, Anonaceae e Euphorbiaceae. No estádio SA, um número muito menor de famílias englobou a grande maioria das espécies recrutadas: Rubiaceae, Monimiaceae, Clusiaceae, Myrtaceae e Lauraceae. Ressalta-se que a diversidade de espécies é também resposta ao tamanho da área amostral, o que em parte pode ter privilegiado o SA.

Entre as plantas que morreram foram identificadas 31 espécies (Tabela 3). Dessas, 25 espécies foram encontradas em formação florestal SM e 19 em SA. Do total de espécies mortas, 11 foram espécies comuns em ambos os estádios. Enquanto no estádio sucessional SM as espécies das famílias que mais contribuíram para o número de plantas mortas foram, respectivamente: Anonaceae, Asteraceae, Melastomataceae e Aquifoliaceae, no SA as espécimes mortas pertenceram, respectivamente, às famílias Rubiaceae e Myrtaceae.
Notadamente nas duas espécies de Asteraceae (Vernonia discolor e Piptocarpha angustifolia) e Cecropia glaziovii, foram observados somente indivíduos mortos nos dois estádios estudados; tampouco foi constatado recrutamento delas (Tabela 3). De modo semelhante, nas espécies Cabralea canjerana, Brosimum lactencens, Zollernia ilicifolia, Maytenus robusta $\mathrm{e}$ várias espécies de Rubiaceae, especialmente as do gênero Psychotria, não foi observada a ocorrência de mortalidade, porém apenas recrutamento, em ambos os estádios. Contudo, as principais espécies que caracterizaram o SA em termos de ocorrência de recrutamento e nenhuma mortalidade foram: Copaiba trapezifolia, Aspidosperma parvifolium, Hirtella hebeclada, Cryptocaria moschata, Endlicheria paniculata e Ocotea catharinensis. No entanto, as espécies exclusivamente recrutadas em SM foram: Sloanea guianensis, Nectandra oppositifolia, Matayba guianensis, Casearia sylvetris, Clethra scabra, Clusia parvifolia, Talauma ovata, Alchornea sidifolia, Hyeronima alchorneoides, Pera glabrata e Miconia cabucu. Por fim, tem-se um grupo de espécies que demonstraram altos índices de mortalidade e relativamente baixos para recrutamento, nos dois estádios. São representantes desse grupo Xylopia brasiliensis, Rollinea sp e Guateria sp.

R. Árvore, Viçosa-MG, v.33, n.1, p.101-115, 2009 
Tabela 3 - Número de plantas arbóreas $\left(\mathrm{ha}^{-1} / \mathrm{ano}\right.$ ) nas categorias recrutadas (PREC), mortas (PMOR) e ramificadas (PRAM) com DAP $\geq 5 \mathrm{~cm}$ por família e espécie no estádio secundário avançado (SA) e no secundário médio (SM), avaliados no período de 1994-2000. São Pedro de Alcântara, SC, Brasil

Table 3 - Number (ha-1/year) of recruited (PREC), dead (PMOR) and branched (PRAM) plants with DBH $\geq 5 \mathrm{~cm}$ by family and species, in forest successional secondary old growth (SA) and secondary medium growth (SM) stages, evaluated from 1994-2000. São Pedro de Alcântara, SC, Brazil

\begin{tabular}{|c|c|c|c|c|c|c|}
\hline \multirow[t]{2}{*}{ Família e espécie } & \multicolumn{2}{|c|}{ PREC } & \multicolumn{2}{|c|}{ PMOR } & \multicolumn{2}{|c|}{ PRAM } \\
\hline & SA & SM & SA & SM & SA & SM \\
\hline Annonaceae & & & & & & \\
\hline Annonaceae sp & 1,2 & & & & & \\
\hline Guateria $\mathrm{sp}$ & & & 3,0 & 16,1 & & \\
\hline Guatteria australis St.-Hil. & & 2,3 & & & & \\
\hline Rolinea exalbida $\mathrm{M}$. & & 4,6 & & & & \\
\hline Rollinia sericea $\mathrm{R}$. E. Fries & & 2,3 & & & & \\
\hline Rollinia ssp & 1,2 & 4,6 & 1,0 & 74,7 & 0,7 & 7,2 \\
\hline $\begin{array}{l}\text { Xylopia brasiliensis Spreng. } \\
\text { Apocynaceae }\end{array}$ & & 4,6 & & 22,2 & & \\
\hline $\begin{array}{l}\text { Aspidosperma parvifolium (Müll.Arg.) A. DC. } \\
\text { Aquifoliaceae }\end{array}$ & 1,2 & & & & 0,7 & \\
\hline Ilex microdonta Reisseck & & 2,3 & & & & \\
\hline $\begin{array}{l}\text { Ilex spp } \\
\text { Asteraceae }\end{array}$ & & & & 12,1 & & \\
\hline Piptocarpha angustifolia Dusén & & & & 12,1 & & \\
\hline Vernonia discolor (Spr.)Less. & & & 1,0 & 10,1 & & \\
\hline Bignoniaceae & & & & & & \\
\hline $\begin{array}{l}\text { Jacaranda micrantha } \text { Cham. } \\
\text { Caesalpiniaceae }\end{array}$ & & 13,7 & & 10,1 & & 9,0 \\
\hline Copaifera trapezifolia Hayne & 1,2 & & & & & \\
\hline Zollernia ilicifolia Vog. & 1,2 & 2,3 & & & & \\
\hline Cecropiaceae & & & & & & \\
\hline $\begin{array}{l}\text { Cecropia glaziovii Snethl. } \\
\text { Celastraceae }\end{array}$ & & & 3,0 & 8,1 & & \\
\hline $\begin{array}{l}\text { Maytenus robusta } \text { Reiss. } \\
\text { Chrysobalanaceae }\end{array}$ & 1,2 & 2,3 & & & 0,7 & 1,8 \\
\hline $\begin{array}{l}\text { Hirtella hebeclada Moricand ex A. DC. } \\
\text { Clethraceae }\end{array}$ & 3,5 & & & & 0,7 & \\
\hline $\begin{array}{l}\text { Clethra scabra (Meissn.) Sleum } \\
\text { Clusiaceae }\end{array}$ & & 9,1 & & 8,1 & & 7,2 \\
\hline Clusia parviflora (Saldanha) Engler & & 13,7 & & 6,1 & & 5,4 \\
\hline $\begin{array}{l}\text { Garcinia gardneriana (Panch. e Triana) Zappi } \\
\text { Cunoniaceae }\end{array}$ & 11,6 & 2,3 & & & & 1,8 \\
\hline $\begin{array}{l}\text { Lamanonia speciosa (Camb.) L.B Smith } \\
\text { Elaeocarpaceae }\end{array}$ & 1,2 & & & & & 1,8 \\
\hline $\begin{array}{l}\text { Sloanea guianensis (Aubl.) Benth. } \\
\text { Euphorbiaceae }\end{array}$ & & 9,1 & 1,0 & 4,0 & 2,9 & 46,9 \\
\hline Alchornea sidifolia Muell. Arg. & & 2,3 & & & & \\
\hline Alchornea triplinervia (Spreng.) M. Arg. & & & & & 1,5 & \\
\hline Hyeronima alchorneoides Fr. Allem. & & 13,7 & & 4,0 & & 21,6 \\
\hline Pera glabrata (Schott) Baill. & & 2,3 & & 4,0 & & 3,6 \\
\hline $\begin{array}{l}\text { Pousandra morisiana (Casar.) Radlk. } \\
\text { Fabaceae }\end{array}$ & 1,2 & & & & & \\
\hline $\begin{array}{l}\text { Andira anthelma (Vell.) Macbr. } \\
\text { Flacourtiaceae }\end{array}$ & & & & & & 5,4 \\
\hline Casearia sylvestris $\mathrm{Sw}$. & & 13,7 & 1,0 & 4,0 & 0,7 & 16,2 \\
\hline
\end{tabular}

R. Árvore, Viçosa-MG, v.33, n.1, p.101-115, 2009 
Tabela 3 - Cont.

Table 3 - Cont.

\begin{tabular}{|c|c|c|c|c|c|c|}
\hline \multirow[t]{2}{*}{ Família e espécie } & \multicolumn{2}{|c|}{ PREC } & \multicolumn{2}{|c|}{ PMOR } & \multicolumn{2}{|c|}{ PRAM } \\
\hline & SA & SM & SA & SM & SA & SM \\
\hline \multicolumn{7}{|l|}{ Lauraceae } \\
\hline Aiouea saligna Meissner & & & & & & 3,6 \\
\hline Cryptocaria moschata $\mathrm{Mez}$ & 1,2 & & & & 4,4 & 1,8 \\
\hline Endlicheria paniculata (Spr.) Macbr. & 1,2 & & & & & \\
\hline Nectandra oppositifolia Nees & & 9,1 & & & & \\
\hline Nectandra spp & 1,2 & 2,3 & 3,0 & 4,0 & 0,7 & 5,4 \\
\hline Ocotea catharinensis $\mathrm{Mez}$ & 2,3 & & & & & \\
\hline Ocotea $\mathrm{ssp}$ & 1,2 & & & & 3,6 & \\
\hline \multicolumn{7}{|l|}{ Magnoliaceae } \\
\hline \multirow{2}{*}{\multicolumn{7}{|c|}{ Melastomataceae }} \\
\hline & & & & & & \\
\hline Miconia сариси Hoehme & & 22,9 & & 4,0 & & \\
\hline Miconia cinnamomifolia (DC) Naudin & & & & 2,0 & & 1,8 \\
\hline Miconia cubatanensis Hoehne & 1,2 & & & & & 3,6 \\
\hline Miconia rigidiuscula Cong. & & & & 2,0 & & \\
\hline Miconia spp & & 4,6 & 1,0 & 2,0 & & \\
\hline Tibouchina sellowiana (Cham.) Cogn. & 1,2 & 2,3 & & 2,0 & & 1,8 \\
\hline Melastomatacea $\mathrm{ssp}$ & & 4,6 & & 2,0 & & \\
\hline \multicolumn{7}{|l|}{ Meliaceae } \\
\hline Cabralea canjerana (Vell.) Mart. & 1,2 & 2,3 & & & 0,7 & 3,6 \\
\hline Trichilia sp. & & 2,3 & & & & \\
\hline Cedrela fissilis Vell. & 1,2 & & 1,0 & 2,0 & & \\
\hline \multicolumn{7}{|l|}{ Mimosaceae } \\
\hline Albizia cf. edwallii (Hoehne) Barneby et J. Grimes & & 4,6 & & & 2,2 & 7,2 \\
\hline Inga sessilis (Vell.) Mart. & 1,2 & & & & & \\
\hline \multicolumn{7}{|l|}{ Monimiaceae } \\
\hline Mollinedia spp & 11,6 & & 4,0 & 2,0 & 4,4 & 5,4 \\
\hline Moraceae & 3,5 & & & & & \\
\hline Brosimum lactescens (S.Moore) Harms & 3,5 & & & & & \\
\hline Coussapoa schottii Miq. & 1,2 & & & & 0,7 & \\
\hline Ficus spp & 1,2 & & & & 0,7 & 1,8 \\
\hline Sorocea bonplandii (Baill.) Burger, Lanj. e Boer & & & & & & 1,8 \\
\hline \multicolumn{7}{|l|}{ Myristicaceae } \\
\hline \multirow{2}{*}{\multicolumn{7}{|c|}{ Myrsinaceae }} \\
\hline & & & & & & \\
\hline Myrcine umbellata Mat. Ex DC. & 1,2 & & & & & \\
\hline Myrsine spp & & & 2,0 & & 0,7 & \\
\hline \multicolumn{7}{|l|}{ Myrtaceae } \\
\hline Calyptranthes grandifolia Berg & & 2,3 & & & & \\
\hline Myrcia rostrata $\mathrm{DC}$. & & 4,6 & & & & 3,6 \\
\hline Myrtaceae ssp & 10,5 & 6,9 & 9,1 & & 0,7 & \\
\hline Marlieria parviflora Berg. & & 2,3 & & & & \\
\hline Psidium cattleianum Sabine & & & & & & 3,6 \\
\hline Nyctaginaceae & & & & & & \\
\hline Guapira opposita (Vell.) Reitz & 5,8 & 4,6 & 4,0 & & 0,7 & 1,8 \\
\hline Ochnaceae & & & & & & \\
\hline Ouratea parviflora (DC.) Baill. & 1,2 & & & & & \\
\hline Olacaceae & & & & & & \\
\hline Heisteria silvianii Scwacke & & & & & 1,5 & \\
\hline
\end{tabular}

R. Árvore, Viçosa-MG, v.33, n.1, p.101-115, 2009 
Tabela 3 - Cont.

Table 3 - Cont

\begin{tabular}{|c|c|c|c|c|c|c|}
\hline \multirow[t]{2}{*}{ Família e espécie } & \multicolumn{2}{|c|}{ PREC } & \multicolumn{2}{|c|}{ PMOR } & \multicolumn{2}{|c|}{ PRAM } \\
\hline & SA & SM & SA & SM & SA & SM \\
\hline \multicolumn{7}{|l|}{ Phytolacaceae } \\
\hline \multicolumn{7}{|l|}{ Quinaceae } \\
\hline $\begin{array}{l}\text { Quiina glaziovii Engl. } \\
\text { Rosaceae }\end{array}$ & 1,2 & & & & & \\
\hline $\begin{array}{l}\text { Prunus cf brasiliensis (Cham. e Schlecht } \\
\text { Rubiaceae }\end{array}$ & & & & & & 1,8 \\
\hline Amaioua guianensis Aubl. & 1,2 & & & & & 1,8 \\
\hline Bathysa meridionalis Smith.e Downs & 2,3 & 16,0 & 2,0 & & 0,7 & 16,2 \\
\hline Posoqueria latifolia (Rudge) R.e S. & 1,2 & 2,3 & & & & \\
\hline Psychotria cf. suterella Müll. Arg. & 11,6 & 2,3 & & & & \\
\hline Psychotria longipes Muell. Arg. & 1,2 & 32,0 & & & & 5,4 \\
\hline Psychotria stenocalyx Müll. Arg. & 1,2 & 2,3 & & & & \\
\hline Psychotria spp & 1,2 & & 9,1 & & 1,5 & \\
\hline $\begin{array}{l}\text { Rudgea jasminoides (Cham.) Müll. Arg. } \\
\text { Rutaceae }\end{array}$ & 11,6 & & 1,0 & & 0,7 & \\
\hline \multicolumn{7}{|l|}{ Sapindaceae } \\
\hline $\begin{array}{l}\text { Matayba guianensis Aublet } \\
\text { Verbenaceae }\end{array}$ & & 25,1 & & & & 5,4 \\
\hline Vitex megapotamica (Spreng.) Mold. & & & & & & 3,6 \\
\hline indeterminadas & 2,3 & 4,6 & 54,5 & 4,0 & & \\
\hline total de plantas/ha/ano & 114 & 272 & 103 & 224 & 32 & 220 \\
\hline total de espécies & 41 & 41 & 25 & 19 & 34 & 22 \\
\hline Qui-quadrado $\left(\chi^{2}\right)$ & $\begin{aligned} \mathrm{P} \\
\left(\chi^{2} 24\right.\end{aligned}$ & $\left.\begin{array}{ll}1 \\
L\end{array} 40\right)$ & $\begin{aligned} & 1 \\
&\left(\chi^{2}\right. 1\end{aligned}$ & $\begin{array}{ll}1 \\
\text { L } 24)\end{array}$ & $\begin{aligned} & \mathrm{P} \\
&\left(\chi^{2}\right. 16\end{aligned}$ & $\begin{array}{ll}01 \\
\text { GL 33) }\end{array}$ \\
\hline
\end{tabular}

Do universo de espécies avaliadas neste trabalho, foi possível constatar que 44 ramificaram abaixo do DAP (Tabela 3). Metade das espécies com ramificações (22) ocorreram apenas no estádio SM e 10 espécies, exclusivamente no SA. Espécies que ocorreram nos dois grupos sucessionais totalizaram 12. As principais espécies que apresentaram comportamento de ramificação foram: Sloanea guianensis, Hyeronima alchorneoides, Casearia sylvestris, Bathysa meridionalis, Molinedia ssp e Albizia cf. edwallii.

A ramificação de plantas abaixo do DAP mostrouse de grande significado biológico na caracterização e diferenciação entre os estádios sucessionais da floresta secundária. Verificou-se que a ramificação está associada à especificidade por espécies. $\mathrm{O}$ fato de ser detectada sete vezes maior a densidade no estádio SM, embora o número das espécies tenha sido um terço inferior comparativamente a $\mathrm{SA}$, evidencia que a ramificação é favorecida pela maior intensidade luminosa ocorrente nesse estádio; ainda se observou que houve grande e constante dinâmica no recrutamento de ramos em plantas ramificadas. Mesmo assim, o número de plantas ramificadas não se alterou em ambos os estádios sucessionais durante os anos de avaliação, uma vez que a alta taxa de mortalidade foi compensada pelo constante recrutamento, o que manteve o número de ramos inalterado durante o período avaliado. Porém, as diferenças apresentadas no número de plantas distribuídas por espécie, entre as duas formações florestais SM e SA (Tabela 3), nas três categorias analisadas, plantas recrutadas, plantas mortas e plantas ramificadas, foram estatisticamente significativas $(\mathrm{P}<0,001)$.

As diferenças entre os DAPs médios dos dois estádios florestais avaliados, tanto para plantas remanescentes quanto para mortas e ramificadas (Tabela 4), foram estatisticamente significativas $(\mathrm{P}<0,05)$. 
No SA, o DAP médio no ano $2000(15,6 \mathrm{~cm})$ foi superior a SM (12 cm). Mesmo assim, independentemente dos estádios, em sete anos de avaliação constatou-se incremento de $1 \mathrm{~cm}$ no valor médio do DAP. Também no estádio SA foram identificados os maiores valores médios de DAP para plantas remanescentes, mortas e ramificadas, comparativamente ao SM. Embora os diâmetros (Tabela 4) e área basal (Tabela 6) tenham sido menores no SM, ainda o incremento diamétrico ou área basal foram muito próximos entre os dois estádios, supõe-se que o dinamismo em ambas as áreas tende para uma similar produção de biomassa florestal.

O maior incremento corrente anual (ICA) em DAP foi observado nas plantas ramificadas, cerca de seis vezes maior do que as plantas remanescentes (Tabela 4).
O ICA das plantas remanescentes foi praticamente igual em ambos os estádios sucessionais. O mesmo não ocorreu nas categorias das plantas recrutadas, mortas e ramificadas, em que os incrementos no SM foram superiores. Supõe-se que no estádio menos avançado a saída de plantas (mortalidade) e, no entanto, a entrada (recrutamento) está fortemente associada ao processo sucessional, visto que os espécimes, ainda que morram, tenham melhores condições de crescimento, em comparação com aquelas que morrem nos estádios mais avançados. E, no entanto, os espécimes recrutados no SM são as espécies colonizadoras de excelência no novo sítio ambiental, o que no estádio SA o ICA inferior pode representar a oportunidade de recrutamento por clareira ou de maior esforço competitivo entre espécimes depositados no banco da regeneração.

Tabela 4 - Médias anuais de DAP médio $(\mathrm{cm})$ seguido do erro-padrão da média $( \pm)$ das categorias de plantas remanescentes (PREM), recrutadas (PREC), mortas (PMOR) e ramificadas (PRAM) e estimativas do incremento corrente anual (ICA, em cm) e teste-t nos estádios sucessionais secundário avançado (SA) e secundário médio (SM) remanescentes da Floresta Ombrófila Densa, avaliados no período de 1994 a 2000. São Pedro de Alcântara, SC, Brasil

Table 4-Annual means of the medium DBH $(\mathrm{cm})$, followed by the standard error of the means $( \pm)$ for the remnant (PREM), recruited (PREC), dead (PMOR) and branched plants below DBH (PRAM) categories, and estimates of the current annual increment (ICA, in cm) and t-test of secondary old growth (SA) and secondary medium growth (SM) successional stages of the Ombrophylus Dense Forest, evaluated from 1994 to 2000. São Pedro de Alcântara, SC, Brazil

\begin{tabular}{lcccccccc}
\hline ano & \multicolumn{2}{c}{ PREM } & \multicolumn{2}{c}{ PREC } & \multicolumn{2}{c}{ PMOR } & \multicolumn{2}{c}{ PRAM } \\
\hline & SA & SM & SA & SM & SA & SM & SA & SM \\
\hline 1994 & 14,50 & 10,98 & - & - & 11,38 & 10,20 & 9,64 & 8,48 \\
& $( \pm 8,40)$ & $( \pm 4,18)$ & & & $( \pm 6,35)$ & $( \pm 4,95)$ & $( \pm 3,62)$ & $( \pm 2,21)$ \\
1995 & 14,68 & 11,21 & 5,92 & 5,83 & 12,57 & 13,80 & 9,97 & 8,73 \\
& $( \pm 8,43)$ & $( \pm 4,22)$ & $( \pm 0,76)$ & $( \pm 0,83)$ & $( \pm 6,51)$ & $( \pm 4,79)$ & $( \pm 3,72)$ & $( \pm 2,29)$ \\
1996 & 14,86 & 11,31 & 5,55 & 5,70 & 21,4 & 8,92 & 10,01 & 8,76 \\
& $( \pm 8,60)$ & $( \pm 4,27)$ & $( \pm 0,71)$ & $( \pm 0,68)$ & $( \pm 6,47)$ & $( \pm 3,82)$ & $( \pm 3,65)$ & $( \pm 2,39)$ \\
1997 & 15,04 & 11,52 & 5,31 & 5,56 & 19,97 & 8,17 & 10,11 & 8,88 \\
& $( \pm 8,68)$ & $( \pm 4,40)$ & $( \pm 0,33)$ & $( \pm 0,42)$ & $( \pm 6,07)$ & $( \pm 3,74)$ & $( \pm 3,79)$ & $( \pm 2,46)$ \\
1998 & 15,10 & 11,58 & 7,24 & 5,95 & 10,31 & 10,75 & 10,48 & 9,09 \\
& $( \pm 8,76)$ & $( \pm 4,45)$ & $( \pm 2,53)$ & $( \pm 1,36)$ & $( \pm 4,71)$ & $( \pm 4,39)$ & $( \pm 4,20)$ & $( \pm 2,64)$ \\
1999 & 15,30 & 11,78 & 5,33 & 5,68 & 10,46 & 7,57 & 10,35 & 9,21 \\
& $( \pm 8,90)$ & $( \pm 4,51)$ & $( \pm 0,68)$ & $( \pm 0,93)$ & $( \pm 4,11)$ & $( \pm 3,35)$ & $( \pm 4,08)$ & $( \pm 2,69)$ \\
2000 & 15,55 & 12,03 & 5,49 & 5,32 & - & - & 10,42 & 9,20 \\
& $( \pm 9,05)$ & $( \pm 4,66)$ & $( \pm 0,55)$ & $( \pm 0,25)$ & & & $( \pm 4,04)$ & $( \pm 2,63)$ \\
\hline média & - & - & $\mathbf{5 , 8 1}$ & $\mathbf{5 , 6 7}$ & $\mathbf{1 4 , 3 5}$ & $\mathbf{9 , 9 0}$ & $\mathbf{1 0 , 1 4}$ & $\mathbf{8 , 9 1}$ \\
\hline teste-t & $18,4^{* *}$ & \multicolumn{7}{c}{$0,4^{\text {ns }}$} \\
\hline ICA (cm) & 0,175 & 0,176 & 0,082 & 0,144 & 0,145 & 0,098 & 1,11 & 1,03 \\
\hline
\end{tabular}

ns: Não-significativo P $>0,05 ; *$ Signific. $\mathrm{P}<0,05 ; \mathrm{e} * *$ Signific. $\mathrm{P}<0,001$. 
A distribuição diamétrica das plantas remanescentes em ambas as formações indicou que mais que $50 \%$ apresentaram DAP $<10 \mathrm{~cm}$ (Tabela 5 e Figura 1). Com o aumento das classes DAP, cresceram as diferenças entre as duas formações. O número de indivíduos com DAP $<20 \mathrm{~cm}$, em SM, foi 89,4\%, enquanto em SA esse valor foi de $77,9 \%$. Notadamente na classe DAP $<30 \mathrm{~cm}$ encontram-se $99,4 \%$ em SM; o restante $(0,6 \%)$ com
DAP $\geq 30 \mathrm{~cm}$ foi representado exclusivamente pelas três espécies: Clethra scabra, Miconia cabucu e Seguieria glaziovii. Em SA, a classe DAP $<30 \mathrm{~cm}$ concentrou $90,3 \%$, sendo o restante $(9,7 \%)$ nas classes de DAP $\geq 30 \mathrm{~cm}$ composto por 39 espécies. Constatouse que, quanto mais avançado for o estádio sucessional da floresta, maiores serão os valores de amplitude diamétrica.

Tabela 5 - Frequência relativa das 10 espécies florestais de maior densidade classificadas em cada classe de DAP e estádio: secundário médio (SM) e secundário avançado (SA) da Floresta Ombrófila Densa, em São Pedro de Alcântara, $\mathrm{SC}, \mathrm{Brasil}$. Entre parênteses é apresentada a frequência relativa

Table 5 - Relative frequency of the 10 species with highest density classified in each each class of DBH and in successional stages of secondary old growth (SA) and secondary medium growth (SM) of the Ombrophylus Dense Forest in São Pedro de Alcântara, SC, Brazil. Relative frequency is presented in parentheses

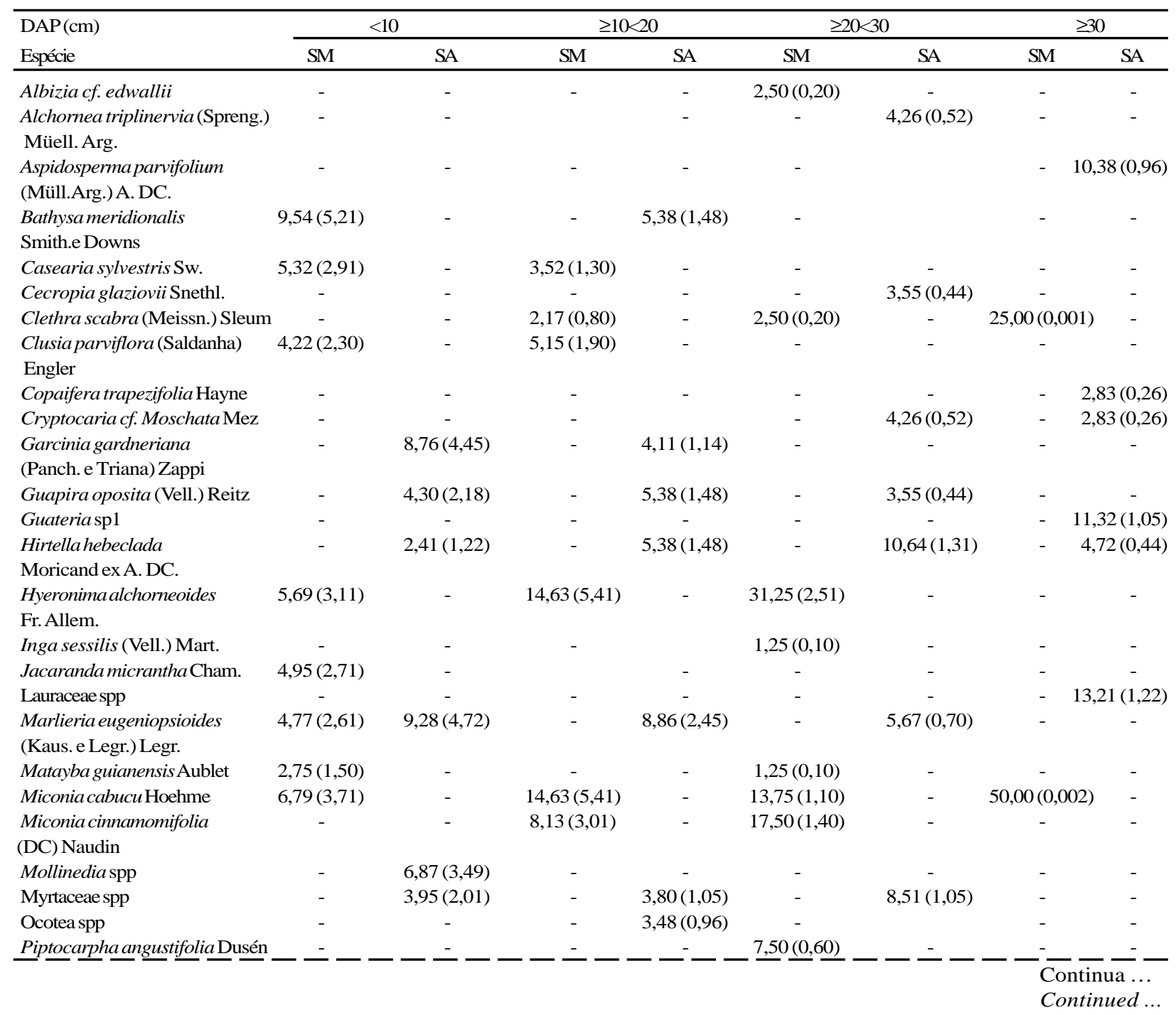

R. Árvore, Viçosa-MG, v.33, n.1, p.101-115, 2009 
Tabela 4 - Cont.

Table 4-Cont.

\begin{tabular}{|c|c|c|c|c|c|c|c|c|}
\hline \multirow{2}{*}{$\begin{array}{l}\mathrm{DAP}(\mathrm{cm}) \\
\text { Espécie }\end{array}$} & \multicolumn{2}{|c|}{$<10$} & \multicolumn{2}{|c|}{$\geq 10<20$} & \multicolumn{2}{|c|}{$\geq 20<30$} & \multicolumn{2}{|c|}{$\geq 30$} \\
\hline & SM & SA & SM & SA & SM & SA & SM & SA \\
\hline Psychotria cf. Suterella Müll. Ar & rg. $\quad-$ & $4,12(2,10)$ & - & - & - & - & - & - \\
\hline $\begin{array}{l}\text { Psychotria longipes } \\
\text { Muell. Arg. }\end{array}$ & $11,19(6,11)$ & - & $13,82(5,11)$ & - & $11,25(0,90)$ & $2,84(0,35)$ & - & $2,83(0,26)$ \\
\hline Psychotria spp & - & $3,44(1,75)$ & - & - & - & - & - & - \\
\hline Rolinea exalbida $\mathrm{M}$. & - & - & $2,71(1,00)$ & - & - & - & - & - \\
\hline $\begin{array}{l}\text { Rudgea jasminoides } \\
\text { (Cham.) Müll. Arg. }\end{array}$ & - & $5,15(2,62)$ & - & - & - & - & - & - \\
\hline Seguieria glaziovii Briq. & - & - & - & - & - & - & $25,00(0,001)$ & 1) \\
\hline Inga sessilis (Vell.) Mart. & - & - & - & & $1,25(0,10)$ & - & - & - \\
\hline $\begin{array}{l}\text { Sloanea guianensis } \\
\text { (Aubl.) Benth. }\end{array}$ & $5,32(2,91)$ & $3,78(1,92)$ & $5,69(2,10)$ & $3,16(0,87)$ & - & $2,84(0,35)$ & $-4,72(0,44)$ & \\
\hline Sloanea spp & - & - & - & $3,48(0,96)$ & - & - & - & \\
\hline Talauma ovata St. Hil. & - & - & - & - & $2,50(0,20)$ & - & - & $1,89(0,17)$ \\
\hline Vernonia discolor (Spr.) Less. & - & - & $2,98(1,10)$ & - & $3,75(0,30)$ & - & - & - \\
\hline Virola bicuhyba Schott & - & & - & $3,16(0,87)$ & - & $4,26(0,52)$ & - & $4,72(0,44)$ \\
\hline Total & $\begin{array}{l}60,55 \\
(33,07)\end{array}$ & $\begin{array}{l}52,06 \\
(26,46)\end{array}$ & $\begin{array}{c}73,44 \\
(27,15)\end{array}$ & $\begin{array}{c}46,20 \\
(12,75)\end{array}$ & $\begin{array}{l}93,75 \\
(7,51)\end{array}$ & $\begin{array}{c}50,35 \\
(6,2)\end{array}$ & $\begin{array}{l}100,00 \\
(0,004)\end{array}$ & $\begin{array}{l}59,43 \\
(5,50) \\
\end{array}$ \\
\hline DR sobre total de plantas ${ }^{1}$ & $\begin{array}{c}53,77 \\
(53,77) \\
\end{array}$ & $\begin{array}{c}51,35 \\
(51,35) \\
\end{array}$ & $\begin{array}{c}35,7 \\
(89,43) \\
\end{array}$ & $\begin{array}{c}26,54 \\
(77,89) \\
\end{array}$ & $\begin{array}{c}9,96 \\
(99,38) \\
\end{array}$ & $\begin{array}{c}12,42 \\
(90,31) \\
\end{array}$ & $\begin{array}{c}0,6 \\
(100) \\
\end{array}$ & $\begin{array}{l}9,69 \\
(100) \\
\end{array}$ \\
\hline espécies observados ${ }^{2}$ & 70 & 85 & 47 & 76 & 15 & 56 & 3 & 39 \\
\hline
\end{tabular}

${ }^{1}$ Inclui todas as espécies encontradas na classe DAP, em porcentagens sobre o total de plantas amostradas. Entre parênteses, porcentagem acumulada da soma das classes de DAP. ${ }^{2}$ Número total de espécies observadas em cada classe de DAP.

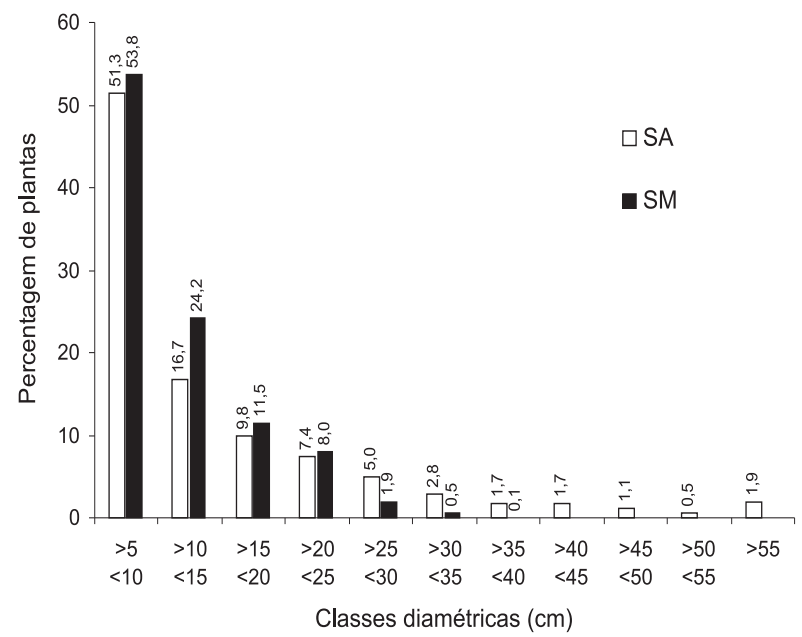

Figura 1 - Distribuição diamétrica da densidade relativa de plantas com DAP $\geq 5 \mathrm{~cm}$, classificadas nos estádios secundários avançado (SA) e secundário médio (SM), em remanescentes da Floresta Ombrófila Densa. São Pedro de Alcântara, SC, Brasil.

Figure 1 - Diameter distribution of the relative density for plants with $D B H \geq 5 \mathrm{~cm}$, classified at the forest successional secondary old growth (SA) and secondary medium growth (SM) stages of the Ombrophylus Dense Forest. São Pedro de Alcântara, SC, Brazil.
No grupo das espécies com DAP $<10 \mathrm{~cm}$, as que mais contribuíram para a densidade de plantas em SM foram, principalmente, Psychotria longipes, Bathysa meridionalis, Miconia cabucu e Hyeronima alchorneoides, e em SA, Marlieria eugeniopsioides, Garcinia gardneriana, Mollinedia spp e Rudgea jasminoides (Tabela 5). Verificouse que, das 10 espécies de maior densidade, dentro de intervalos de classes de DAP, apenas três (Marlieria eugeniopsioides, Psychotria longipes e Sloanea guianensis) foram comuns em pelo menos um intervalo de DAP em ambas as formações florestais. Entre as classes diamétricas foi possível identificar grupos de espécies que caracterizam cada estádio sucessional. Como exemplo, na classe DAP $<10 \mathrm{~cm}$, no SM foram identificadas espécies que participaram do dossel, enquanto no SA as espécies foram exclusivas de subosque florestal. Tal fato evidencia que, no estádio SM, a densidade superior de plantas e maiores taxas de mortalidade pode estar fortemente associada ao estádio evolutivo da sucessão florestal, sendo as espécies pioneiras, já no final do ciclo vital ou na regeneração, substituídas por espécies mais adaptadas ao ambiente sombreado e úmido.

A área basal total na formação florestal SM no ano 2000 foi de $25,6 \mathrm{~m}^{2} \mathrm{ha}^{-1}$, aproximadamente um quarto inferior a SA, que foi 33,3 $\mathrm{m}^{2}$ ha $^{-1}$ (Tabela 6). A média de área basal

R. Árvore, Viçosa-MG, v.33, n.1, p.101-115, 2009 
das plantas recrutadas foi praticamente duas vezes menor que a área basal das plantas mortas. Porém, apesar do balanço negativo entre plantas recrutadas e mortas, o balanço final na área basal foi compensado pelo crescimento diamétrico das remanescentes, que por sua vez proporcionaram um incremento médio de aproximadamente $0,7 \mathrm{~m}^{2} \mathrm{ha}^{-1}$, anualmente, à comunidade florestal. Assim, em seis anos se observou um incremento de $4 \mathrm{~m}^{2}$, em ambos os estádios.

Embora, não fossem observadas diferenças nos valores de incremento anual em área basal entre os dois estádios, ainda que foram observadas diferenças sobre a densidade de plantas e área basal, o crescimento florestal estimado no período de seis anos, em termos de incremento, foram praticamente iguais nos dois estádios florestais. Veloso e Klein (1957), estudando cinco remanescentes primários da Floresta Ombrófila Densa em Brusque, SC, observaram amplitude de 30,9 a 64,7 $\mathrm{m}^{2} \mathrm{ha}^{-1}$ nos valores em área basal. Também, Wernecket al. (2000) observaram forte o dinamismo em formação secundária, com mudanças na distribuição das classes diamétricas, no grupo das espécies pioneiras, e detectaram decréscimo no número de indivíduos.

As 10 famílias com maior densidade em plantas representaram 77,8\% em SM e 65,7\% em SA (Figura 2). Em SM, as famílias que sobressaem e que caracterizam, em termos de densidade, o estádio sucessional foram Melastomataceae, Euphobiaceae e Rubiaceae. Já no estádio SA foram destacadas, principalmente, as famílias Rubiaceae, Myrtaceae e Lauraceae. Contudo, a dominância em densidade principalmente dessas 10 famílias ilustradas na Figura 1 é bem contrastante entre os dois estádios sucessionais avaliados. É relevante perceber que a participação das 10 espécies dominantes na composição florística de cada estádio marca a diferenciação entre os dois estádios sucessionais. Também no Tabela 3 mostrase que qualitativamente a estrutura florística nos diferentes estádios pode ser caracterizada pela exclusividade de espécies ou, mesmo, por famílias presentes ou ausentes nesses estádios. A exclusividade de algumas famílias detectadas apenas em um dos estádios, a exemplo de Monimiaceae, Myristicaceae e entre várias outras, indica que grupos de espécies tem grande funcionalidade na reconstrução da floresta. Isso indica a importância do conhecimento ecológico e a ecologia das espécies para a sua aplicabilidade nos empreendimentos de recuperação de áreas degradadas e, ou, cobertura vegetacional nativa.

As diferenças observadas entre os SM e SA caracterizam a existência de dois ambientes com diferenças no estádio sucessional. Esse fato colabora em classificar o SM em fase sucessional mais inicial, enquadrandose, conforme classificação adotada por Klein (1980), na fase do Miconietum, caracterizada pela dominância da espécie Miconia cinnamomifolia. De fato, no SM foi observada alta dominância dessa espécie. No entanto, o SA encontra-se em estádio mais avançado, podendo ser caracterizado como fase de Mata Secundária (KLEIN, 1980). Contudo, segundo a Resolução nº 04/1994, do CONAMA, ambos os estádios (SM e SA) se enquadraram no estádio avançado de regeneração, permitindo que um estádio sucessional com presença de espécies arbóreas, com predominância do grupo ecológico das pioneiras, fosse enquadrado na mesma fase sucessional de áreas com presença de espécies de um grupo ecológico mais avançado (climácicas).

Tabela 6 - Valores de área basal das espécies arbóreas das categorias de plantas remanescentes (PREM), recrutadas (PREC), mortas (PMOR) e ramificadas (PRAM) e estimativas do incremento corrente anual (ICA, em $\mathrm{cm}$ ) nos estádios sucessionais secundário avançado (SA) e secundário médio (SM), remanescentes da Floresta Ombrófila Densa, avaliados no período de 1994 a 2000. São Pedro de Alcântara, SC, Brasil

Table 6 - Values of basal area for remnant (PREM), recruited (PREC), dead (PMOR) and branched (PRAM) plant categories, and estimates of the current annual increment (ICA, in cm) of secondary old growth (SA) and secondary medium growth (SM) successional stages, Ombrophylus Dense Forest, evaluatied from 1994 to 2000. São Pedro de Alcântara, SC, Brazil

\begin{tabular}{|c|c|c|c|c|c|c|c|c|c|c|}
\hline \multirow[b]{2}{*}{ Ano } & \multicolumn{2}{|c|}{ PREM } & \multicolumn{2}{|c|}{ PREC } & \multicolumn{2}{|c|}{ PMOR } & \multicolumn{2}{|c|}{ PRAM } & \multicolumn{2}{|c|}{ Total } \\
\hline & SA & $\mathrm{SM}$ & SA & SM & SA & SM & SA & $\mathrm{SM}$ & SA & SM \\
\hline 1994 & 28,67 & 20,08 & - & - & - & - & 0,40 & 1,12 & 29,07 & 21,20 \\
\hline 1995 & 29,17 & 20,85 & 0,11 & 0,29 & 0,42 & 0,58 & 0,43 & 1,20 & 29,71 & 22,34 \\
\hline 1996 & 29,98 & 21,13 & 0,04 & 0,20 & 0,38 & 0,83 & 0,43 & 1,21 & 30,45 & 22,54 \\
\hline 1997 & 30,70 & 22,12 & 0,02 & 0,04 & 0,14 & 0,19 & 0,44 & 1,25 & 31,16 & 23,41 \\
\hline 1998 & 31,03 & 22,40 & 0,11 & 0,02 & 0,36 & 0,10 & 0,49 & 1,32 & 31,63 & 23,74 \\
\hline 1999 & 31,78 & 23,15 & 0,04 & 0,14 & 0,28 & 0,42 & 0,47 & 1,37 & 32,29 & 24,66 \\
\hline 2000 & 32,83 & 24,25 & 0,03 & 0,05 & 0,22 & 0,29 & 0,47 & 1,35 & 33,33 & 25,65 \\
\hline Média & - & - & 0,06 & 0,12 & 0,30 & 0,40 & 0,01 & 0,04 & - & - \\
\hline $\mathrm{ICA}$ & 0,69 & 0,70 & - & - & - & - & - & - & 0,71 & 0,74 \\
\hline
\end{tabular}

R. Árvore, Viçosa-MG, v.33, n.1, p.101-115, 2009 


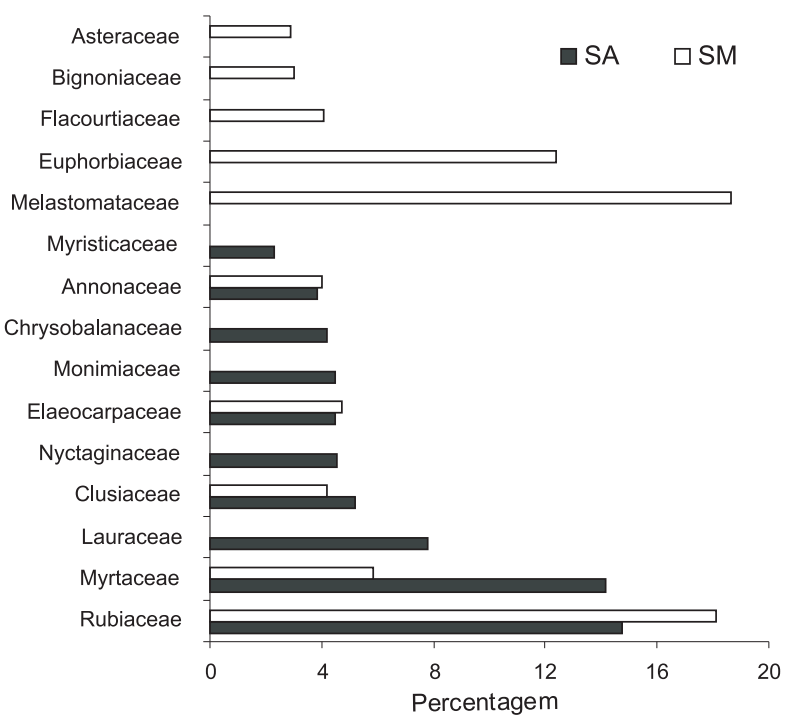

Figura 2 - Densidade relativa das 10 famílias de maior densidade, classificadas nos estádios secundários avançado (SA) e secundário médio (SM), remanescentes da Floresta Ombrófila Densa. São Pedro de Alcântara, $\mathrm{SC}$, Brasil.

Figure 2 -Relative density of the 10 families with highest density, classified at the forest successional secondary old growth (SA) and secondary medium growth (SM) stages of the Ombrophylus Dense Forest. São Pedro de Alcântara, SC, Brazil.

\section{CONCLUSÕES}

Verificou-se que o dinamismo florestal foi qualitativa e quantitativamente caracterizado pela composição florística das espécies recrutadas, mortas e grupo das dominantes. Entre as várias comparações para as formações florestais dos estádios secundários médio (SM) e secundário avançado (SA), destacam-se:

- Várias das espécies que morreram não foram as mesmas que as recrutadas, ou as proporções entre dessas mostram evidência clara de declínio e, ou, ascensão da população da espécie na comunidade florestal em cada estádio sucessional.

- Comparativamente ao SM, o número de espécies no SA foi um terço superior, e proporção similar foi observada nas espécies mortas e ramificadas.

- Comparativamente ao SA, a densidade de plantas foi superior no SM; nas categorias a comunidade superou (60\%); na mortalidade e recrutamento (superior a 100\%); e nas plantas ramificadas a diferença foi exponencialmente superior $(680 \%)$.
- Recrutamento de espécies em SA é composto praticamente de climácicas, e no SM são climácicas e pioneiras.

- Mortalidade de espécies em SM foi caracterizada por espécies pioneiras e, no SA, atingiu principalmente representantes de espécies climácicas (sub-bosque).

- As taxas de incremento corrente anual foram similares entre SA e SM.

- A produção de biomassa florestal mensurada pelo incremento da área basal foi similar entre SA e SM.

No entanto, as informações florísticas indicaram que os remanescentes florestais secundários da Floresta Ombrófila Densa em Santa Catarina encontram-se em crescimento e contínuo dinamismo sucessional, e espécies climáxicas vêm substituindo paulatinamente as espécies construtoras e elevando a diversidade de espécies e a biomassa florestal, evidenciando também, perfeitamente, a funcionalidade dos grupos ecológicos nessa tipologia florestal.

\section{AGRADECIMENTOS}

Ao Conselho Nacional de Desenvolvimento Científico e Tecnológico (CNPq-Brasil), pelas bolsas concedidas aos autores; ao Professor Ademir Reis (Universidade Federal de Santa Catarina, Centro de Ciências Biológicas, Departamento de Botânica) e ao Marcos Eduardo Guerra Sobral (Universidade Federal de Minas Gerais, Instituto de Ciências Biológicas, Departamento de Botânica), pela identificação do material botânico; ao Núcleo de Pesquisa em Florestas Tropicais (NPFT), pelo suporte de material e recursos humanos; e ao FNMA, pelo apoio no período de 1994 a 1996.

\section{REFERÊNCIAS}

\section{Beiguelman, B. Curso prático de}

bioestatística. 2.ed. Ribeirão Preto: Sociedade Brasileira de Genética, 1991.

BUDOWSKI, G. The distinction between old secondary and climax species in tropical central American lowland forest. Turrialba, v.15, n.1, p.40-42, 1965.

BUDOWSKI, G. Distribution of tropical American rain forest species in the light of successional processes. Tropical Ecology, v.11, n.1,p.44-48, 1970.

R. Árvore, Viçosa-MG, v.33, n.1, p.101-115, 2009 
CONSELHONACIONALDOMEIOAMBIENTECONAMA. Resolução CONAMA n.10, de 01 de outubro de 1993 e Resolução n.004, de 04 de maio de 1994. Brasília: 1994.

DALLING, J. W.; STEPHEN, P. H.; SILVEIRA, K. Seed dispersal, seedling establishment and gap partitioning among tropical pioneer trees.

Journal of Ecology, v.86, p.674-689, 1998.

FERREIRA, R. L. C.; SOUZA, A. L.; JESUS, R. M. Dinâmica da estrutura de uma floresta secundária de transição: II-distribuição diamétrica. Revista Árvore, v.22, n.3, p.311-344, 1998.

FONSECA, R. C. B.; RODRIGUES, R. R.

Structural analysis and aspects of the successional mosaico a semi-deciduous forest, in Botucatu (São Paulo State, Brazil). Scientia Forestalis, v.57, n.1, p.27-43, 2000.

FUNDAÇÃO SOS MATAATLÂNTICAEINSTITUTO NACIONALDE PESQUISAS ESPACIAIS, INSTITUTO SOCIOAMBIENTAL. Atlas da evolução dos remanescentes florestais e ecossistemas associados no domínio da Mata Atlântica no período 2000-2005. São Paulo - SP. Disponível em: <http://mapas.sosma.org.br- Atlas da Mata Atlantica-Relatorio2000-2005.pdf.> Acessado em: Agosto de 2008.

INSTITUTO BRASILEIRO DE GEOGRAFIA E ESTATÍSTICA - IBGE. Geografia do Brasil

- Região Sul. Rio de Janeiro: 1990. v.2.

JANZEN, D. H. Herbivores and the number of tree species in tropical forests. The American Naturalist, v.104, n.40, p.501-28, 1970.

KLEIN, R. M. Mapa fitogeográfico do Estado de Santa Catarina. Itajaí: Herbário Barbosa Rodrigues; Florianópolis: Universidade Federal de Santa Catarina, 1978.

KLEIN, R. M. Ecologia da flora e vegetação do Vale do Itajai. Sellowia, v.32, p.165-389, 1980.

KREBS, C .J. Ecological methodology. New York: Harper and Row, 1989.

MAGURRAN, A. E. Ecological diversity and its measurement. New Jersey: Princeton University Press, 1988.

R. Árvore, Viçosa-MG, v.33, n.1, p.101-115, 2009
MANTOVANI, M. et al. Fenologia reprodutiva de espécies arbóreas em uma formação secundária da Floresta Atlântica. Revista Árvore, v.27, n.4, p.451-458, 2003.

MANTOVANI, M. et al. Diversidade de espécies e estrutura sucessional de uma formação secundária da Floresta Ombrófila Densa. Scientia forestalis, v.67, p.14-26, 2005.

NASCIMENTO, H. E. M. et al. Estrutura e dinâmica de populações arbóreas de um fragmento de Floresta Estacional Semidecidual na região de Piracicaba, SP. Revista Brasileira de Biologia, v.59, p.329-342, 1999.

OLIVEIRA FILHO, A. T.; MELLO, J. M.; SCOLFORO, J. R. S. Effects of past disturbance and edges on tree community structure and dynamics within a fragment of tropical semideciduous forest in south-estern Brazil over a five-year period (1987-1992). Plant Ecology, v.131, n.1, p.45-66, 1997.

PAGANO, S. N.; LEITÃO FILHO, H. F.;

CAVASSAN, O. Variação temporal da composição florística e estrutura fitossociológica de uma Floresta Mesófila Semidecídua, Rio Claro, Estado de São Paulo. Revista Brasileira de Biologia, v.55, p.241-258, 1995.

PETRERE, M.; GIORDANO, L. C.; MARCO JR., P. Empirical diversity index applied to Forest communities in different successional stages.

Brazilian Journal of Biology, v.64, p.841851, 2004.

PHILliPS, O. L.; NUNEZ, P.; TIMANA, M. E. Tree mortality and collecting botanical vouchers in tropical forests. Biotropica, v.30, p.298-305, 1998.

Queiroz, M. H. Approche

phytoécologoque et dynamique de formation végetales secondaires dévelopées après aband dês activités agricoles, dans le domaine de la Fôret Ombrophile Dense de versant (Fôret Atlantique) à Santa Catarina - Brésil. 1994. 251f. Tese (Doctor emForest Science) École Nationale du Génie Rural, des Eaux et des Forests, Nancy, França, 1994. 
ROLIM, S. G.; COUTO, H. T. Z.; JESUS, R. M.

Mortalidade e recrutamento de árvores na Floresta Atlântica em Linhares (ES). Scientia

Forestalis, v.55, p.49-69, 1999.

STEENIS van, C. G. G. L. Rejuvenation as a factor for judging the status of vegetation types - the biological nomad theory. In: STUDY OF TROPICAL VEGETATION. KANDY SYMPOSIUM, 1958, Ceylon. Proceedings... Paris: Unesco, 1958. p.212-215.

TABARELLI, M.; MANTOVANI, W. Ocupação de clareiras naturais na floresta na Serra da CantareiraSP. Naturalia, v.22, n.1, p.89-102, 1997.

TILMAN, D. Competition and biodiversity in spatially structured habitats. Ecology, v.75, n.1, p.2-16, 1994.
VELOSO, H. P.; KLEIN, R. M. As comunidades e associações vegetais da Mata Atlântica do Sul do Brasil. I. As comunidades do Município de Brusque, Estado de Santa Catarina. Sellowia, v.8, p.81-235, 1957.

WERNECK, M. S.; FRANCESCHINELLI, E. V.; TAMEIRÃO NETO, E. Mudanças na florística e estrutura de uma Floresta Decídua durante um período de quatro anos (1994-1998), na região do Triângulo Mineiro, MG. Revista Brasileira de Botânica, v.23, n.4, p.401-413, 2000.

WHITMORE, T. C. Secondary succession from seed in Tropical Rain Forests. Forestry Abstracts, v.44, n.12, p.767-779, 1983. 